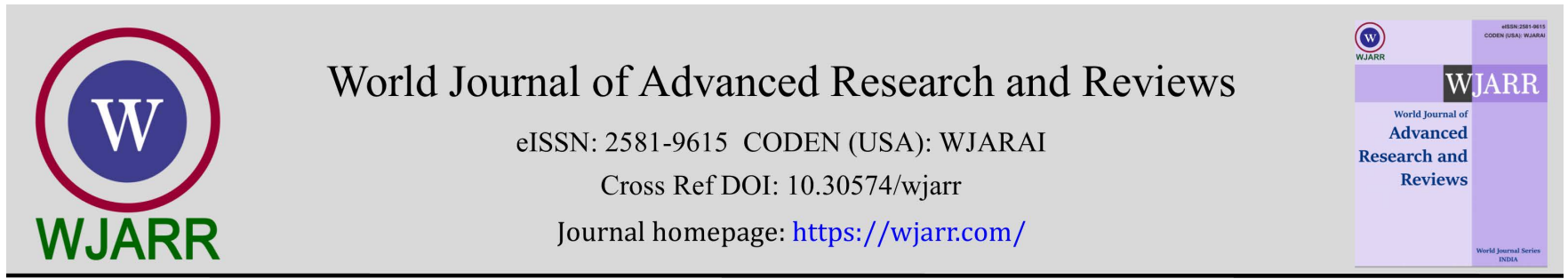

(RESEARCH ARTiClE)

Check for updates

\title{
A study to investigate the difference in functional activity, gait, and balance of stroke patients according to treatment methods
}

Jung-Ho Lee*

Department of Physical Therapy, Kyungdong University: 815, Gyeonhwon-ro, Munmak-eup, Wonju-si, Gangwon-do, Republic of Korea.

World Journal of Advanced Research and Reviews, 2022, 13(02), 082-089

Publication history: Received on 27 December 2021; revised on 03 February 2022; accepted on 05 February 2022

Article DOI: https://doi.org/10.30574/wjarr.2022.13.2.0117

\begin{abstract}
Purpose: This study was conducted to investigate the effect of therapeutic interventions in the field of physical therapy on the improvement of functional activity and gait and balance ability of the upper and lower extremities.

Methods: Twenty subjects of the study participated in the study after hearing the explanation of the study, and were divided into Experimental Group 1 and Experimental Group 2 using a randomization method to participate in the study. Experimental group 1 received functional electrical stimulation treatment for the upper extremities and lower extremities for 20 minutes before PNF, and experimental group 2 received taping therapy to the upper extremities and lower extremities before PNF. In this study, FMA was used to evaluate the functional activity of the subject, walking ability was evaluated through TUG test, and balance was evaluated using BBS.
\end{abstract}

Results: In the results of this study, there was a statistically significant difference in functional activity, gait, and balance between the pre- and post-evaluations. However, no statistically significant difference was found in the analysis comparing the size of the treatment effect between the two experimental groups.

Conclusion: In conclusion, functional electrical stimulation therapy and taping therapy are therapeutic methods that are helpful in improving functional activity, gait, and balance ability in stroke patients. In addition, based on the results of this study, the necessary treatment can be selectively applied according to the patient's situation.

Keywords: Functional electrical stimulation; Taping; Ability; Stroke; Gait; Balance

\section{Introduction}

Hemiplegia is caused by stiffness on the contralateral side of the damaged brain and is a common symptom in stroke patients. Spasticity refers to increased muscle tone than normal in which spasticity, convulsions, hypertonia, and deep tendon hyper reflexes occur due to upper motor neuron lesions. Stroke patients have reduced balance ability due to muscle weakness, changes in muscle tone, sensory impairment, abnormal postural response, and cognitive problems, as well as decreased mobility of the ipsilateral upper and lower extremity joints including the trunk [1].

In stroke patients, damage to the cortex and subcortical areas causes left-right imbalance of the body and creates an asymmetrical posture, making it difficult to maintain the center of the body. Stroke patients have twice as much postural sway compared to normal people, and the weight is excessively supported on the unaffected leg, and an asymmetrical posture is created [2].

\footnotetext{
* Corresponding author: Jung-Ho Lee

Department of Physical Therapy, Kyungdong University: 815, Gyeonhwon-ro, Munmak-eup, Wonju-si, Gangwon-do, Republic of Korea.
}

Copyright $(2022$ Author(s) retain the copyright of this article. This article is published under the terms of the Creative Commons Attribution Liscense 4.0. 
The ability to maintain balance and posture in space is the most basic element in all movements of a person, and in order to do this properly, posture control is required to respond to various environments. Postural control of the human body consists of feed-forward, which occurs before voluntary movement, and feedback, which is actually responsible for postural sway, which occurs after the movement has taken place. Proactive posture control functions to minimize the risk of loss of balance by activating the trunk and leg muscles prior to body agitation and adjusting the position of the body's center of gravity [3].

Treatments based on the theory of motor control re-learning, which allow stroke patients to learn motor functions by creating an environment so that they can more actively use the paralyzed upper and lower extremities for functional movements, are being emphasized. Functional electrical stimulation therapy is a treatment that helps patients maximize motor re-learning during active and repetitive movement training. It is widely used for the purpose of strengthening muscle strength, re-educating muscles, and improving paralyzed limb functions by applying to stroke and spinal cord injury patients [4].

Shoulder joint pain in stroke patients can lead to functional activity limitation due to balance disorders and muscle range movement disorders, so active treatment for shoulder joint pain is required. Also, among the sensory disturbances occurring in stroke patients, proprioception impairment causes muscle and joint ataxia, resulting in loss of balance and inappropriate movements [5].

Taping therapy promotes muscle contraction and relaxation by attaching an elastic adhesive tape with a rate of elasticity similar to that of human muscle to the skin based on exercise muscle physiology and kinematics, and promotes blood circulation to harmonize muscles that are not in harmony with their surroundings. Currently, taping therapy has been applied to prevent injuries in sports players or to support muscles, but recently, it has been applied to improve the range of motion, joint function, movement and discomfort of ordinary people [6].

Therefore, the purpose of this study is to investigate the effects of functional electrical stimulation therapy and taping therapy on functional activity, gait, and balance in stroke patients, and to find out the effects to present the rationale for clinical treatment.

\section{Material and methods}

\subsection{Subjects}

The subject of this study was a convenience sample based on the following selection criteria for stroke patients admitted to a nursing hospital. The study subjects were patients diagnosed with stroke by a specialist, able to communicate, understood the purpose of the study, and agreed to participate in the study in writing (Figure 1).

The selected study subjects were divided into experimental group $1(n=10)$ and experimental group 2 (n=10) by randomization method, functional electrical stimulation treatment was applied to experimental group 1, and taping therapy was applied to experimental group 2.

Subjects voluntarily participated in the study after hearing sufficient explanations about the contents and purpose of this study, experimental procedure, and stability of the study, and participated in the experiment after obtaining written consent to participate in the study. In addition, all processes of this study were conducted in accordance with the guidelines of the Research Ethics Committee and the Declaration of Helsinki.

In this study, experimental group 1 received PNF treatment for 30 minutes during neurodevelopmental treatment, and functional electrical stimulation treatment was applied to paralyzed upper and lower extremities for 20 minutes before PNF treatment.

Experimental group 2 applied the taping therapy to the paralyzed upper and lower extremities before PNF treatment, and received PNF treatment for 30 minutes after the taping was applied. In this study, the pre-evaluation was performed before the application of therapeutic intervention and the post-evaluation was performed after the last treatment. All groups received a total of 18 therapeutic interventions, 3 times a week for 6 weeks. 


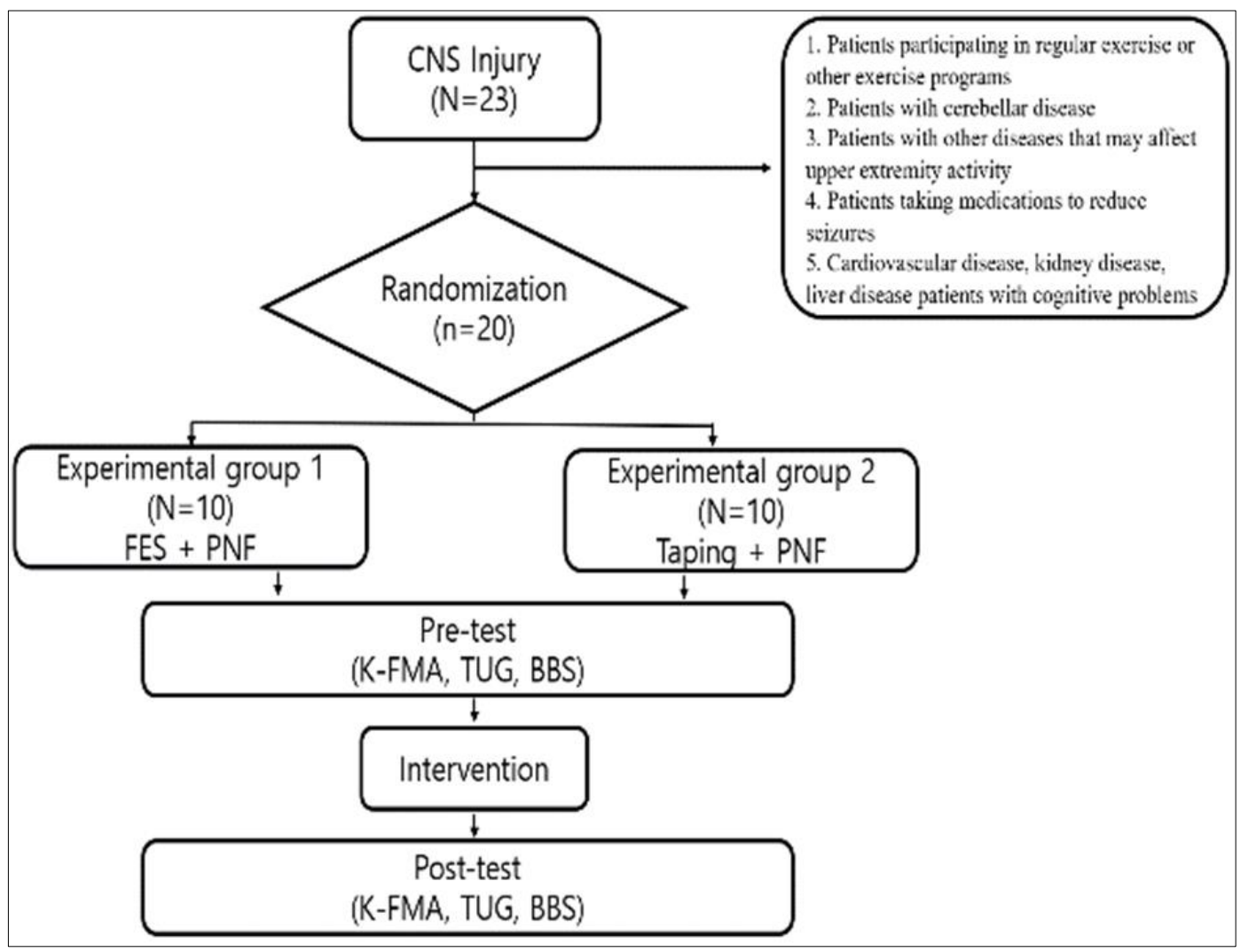

Figure 1 Flow chart

\subsection{Criteria for selection and exclusion of research subjects}

The selection criteria for this study are as follows.

- A patient who understands the content of the study and is able to follow the instructions of the therapist

- $\quad$ Patients not taking psychotropic drugs

- Patients with no vestibular abnormalities

- $\quad$ Patients with no vision or hearing problems

- Patients who have not undergone orthopedic surgery within 3 months

- Patients who can walk with or without braces

The criteria for subject exclusion in this study are as follows.

- $\quad$ Patients participating in regular exercise or other exercise programs

- $\quad$ Patients with cerebellar disease

- Patients with other diseases that may affect upper extremity activity

- $\quad$ Patients taking medications to reduce seizures

- Cardiovascular disease, kidney disease, liver disease patients with cognitive problems

\section{Evaluation method}

\subsection{Korean version of Fugl-Meyer Assessment}

In this study, korean version of Fugl-Meyer assessment was used to evaluate the stages of functional recovery in stroke patients. korean version of Fugl-Meyer assessment was used to evaluate the current patient's motor function or balance status in patients participating in this study and to determine how much improvement has been achieved since.

korean version of Fugl-Meyer assessment mainly consists of tests focusing on motor function or the patient's current movement rather than sensory function, and consists of evaluation items focusing mainly on upper extremity function 
rather than lower extremity function. The Fugl-Meyer assessment consists of a total of 113 questions and a total of 226 points on a 3-point scale. Accordingly, in this study, only the upper extremity Fugl-Meyer assessment terminal items were selected to evaluate the wrist and hand.

\subsection{Timed up and go test}

In this study, the timed up and go test was used to evaluate the subjects' gait. The timed up and go test was developed to assess dynamic balance and mobility. Assessment begins with the patient sitting comfortably in a firm chair. Following the evaluator's verbal instructions, the patient stands up, walks a distance of $3 \mathrm{~m}$ at normal walking speed, and returns to the chair.

In this test, a score within 10 seconds means normal, and a score between 11 seconds and 20 seconds means that you have some degenerative or individual lesions. If it is longer than 30 seconds, it means that there is a high risk of functional mobility impairment and falls.

\subsection{Berg balance scale}

In this study, the Berg balance scale was used to evaluate the balance ability of the subjects, and the Berg balance scale test is a method that can objectively evaluate the static balance ability and the dynamic balance ability in the posture to which the movements of daily life are applied. The Berg balance scale consists of a total of 14 items, with a total score of 0-56, using 0-4 points for each item. A Berg balance score of 41-56 means that independent activities of daily living are possible, and a score of 21-40 means that the balance ability decreases and the risk of a fall increases, so caution is required. In addition, a score of 20 or less means that the balance ability is excessively deteriorated, making walking and functional activities difficult, and the risk of a fall is very high.

\section{Treatment methods}

\subsection{Functional electrical stimulator}

In order to apply functional electrical stimulation in this study, a functional electrical stimulator (Microstim, German) that can control frequency, contraction time, relaxation time, and energization time was used.

An active electrode was placed $1 \mathrm{~cm}$ below the peroneal head to selectively stimulate the peroneal nerve, and the reference electrode was placed on the anterior tibial muscle about $10 \mathrm{~cm}$ below the peroneal head to stimulate the ankle joint and foot muscles innervated by the peroneal nerve. The intensity of the stimulation current was applied at a sufficient intensity for dorsiflexion and valgus to occur during energization according to the response of the ankle joint to the stimulus.

In addition, to stimulate the wrist and finger extensors, the active electrode of functional electrical stimulation was placed $1 \mathrm{~cm}$ below the outer side of the radial head, and the basic electrode was placed $3 \mathrm{~cm}$ above the back of the wrist joint. And electrical stimulation was applied within the range where the patient did not complain of pain. Functional electrical stimulation treatment was applied for 20 minutes before the proprioceptive neuromuscular facilitation method was applied, and was applied 3 times a week, a total of 18 times for 6 weeks.

\subsection{Taping therapy}

In this study, elastic tape (Benefact, Japan) was used for taping application.

For the lower extremity taping method, an elastic tape with a width of $5 \mathrm{~cm}$ and a length of $40 \mathrm{~cm}$ was used on the affected tibialis anterior muscle. One end was fixed between the big toe and the index toe of the instep, and with the ankle plantar-flexed, tape was attached to the outside of the knee along the direction of the muscle movement.

The taping therapy for the extensor muscle of the wrist was applied to the medial and lateral parts of the medial epicondyle of the humerus, starting from the wrist joint and ascending along the direction of the muscle.

\section{Statistical analysis method}

For statistical analysis, SPSS version 18.0 statistical processing program was used. Data were expressed as mean and standard deviation, and homogeneity of the dependent variables was tested. A paired t-test was performed for comparative analysis of pre-evaluation and post-evaluation within the experimental group, and an independent $t$-test 
was performed to examine the difference in treatment effect between the experimental groups. The statistical significance level $\alpha$ was set to 0.05 .

\section{Results}

In the results of the FMA wrist evaluation conducted to investigate the functional activity of stroke patients, there was a statistically significant within-group difference in both Experimental Group 1 and Experimental Group 2. However, there was no statistically significant difference in the comparison of the treatment effect between the experimental groups on the FMA wrist (figure 2).

There was a significant difference in all groups in the statistical analysis of the pre- and post-evaluation of the FMA hand. However, there was no statistically significant difference in the comparison of the treatment effect between the experimental groups on the FMA hand (figure 3).

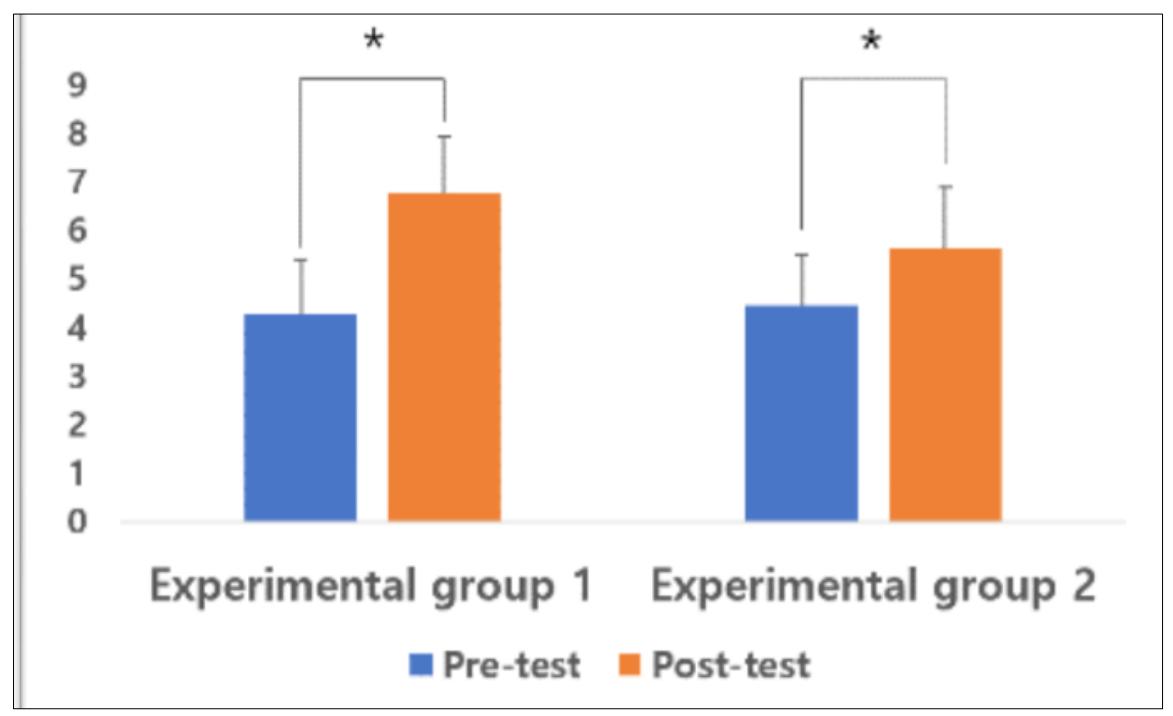

Figure 2 Changes in FMA wrist scores according to treatment methods

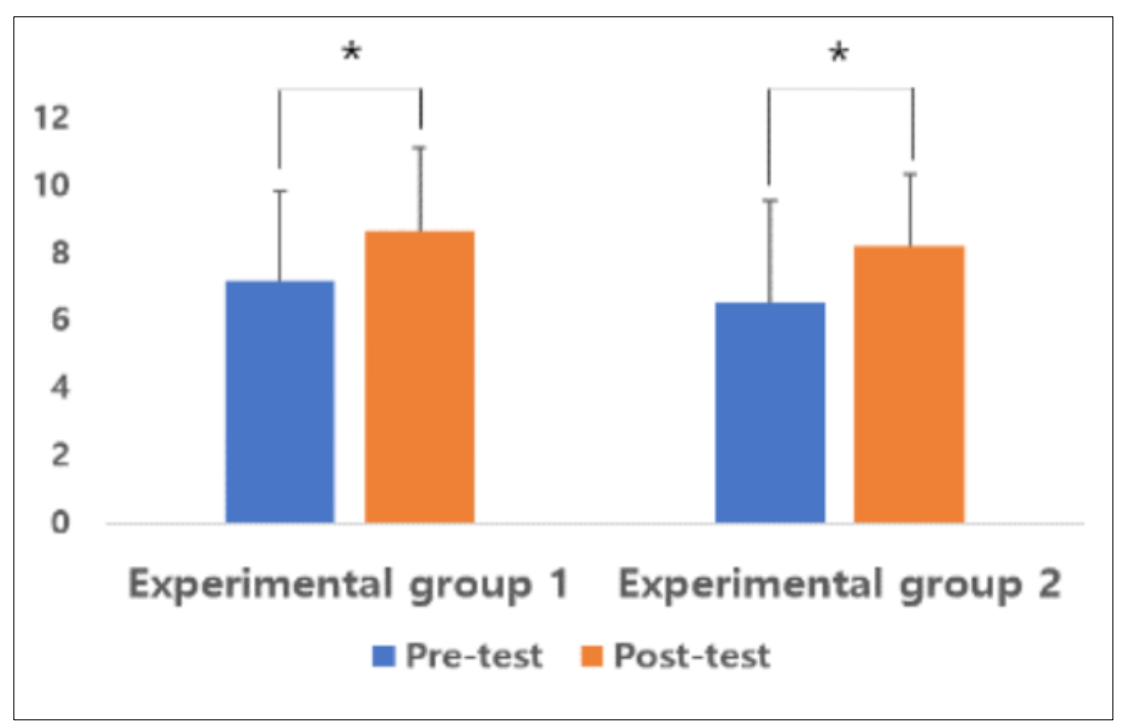

Figure 3 Changes in FMA hand scores according to treatment methods

In the TUG test conducted to examine the gait ability of the patient, there was a statistically significant difference in the increase in walking speed in both the experimental group 1 and the experimental group 2 in the post-evaluation 
compared to the pre-evaluation. However, there was no statistically significant difference in the comparison of the treatment effect between the experimental groups on the gait ability (figure 4).

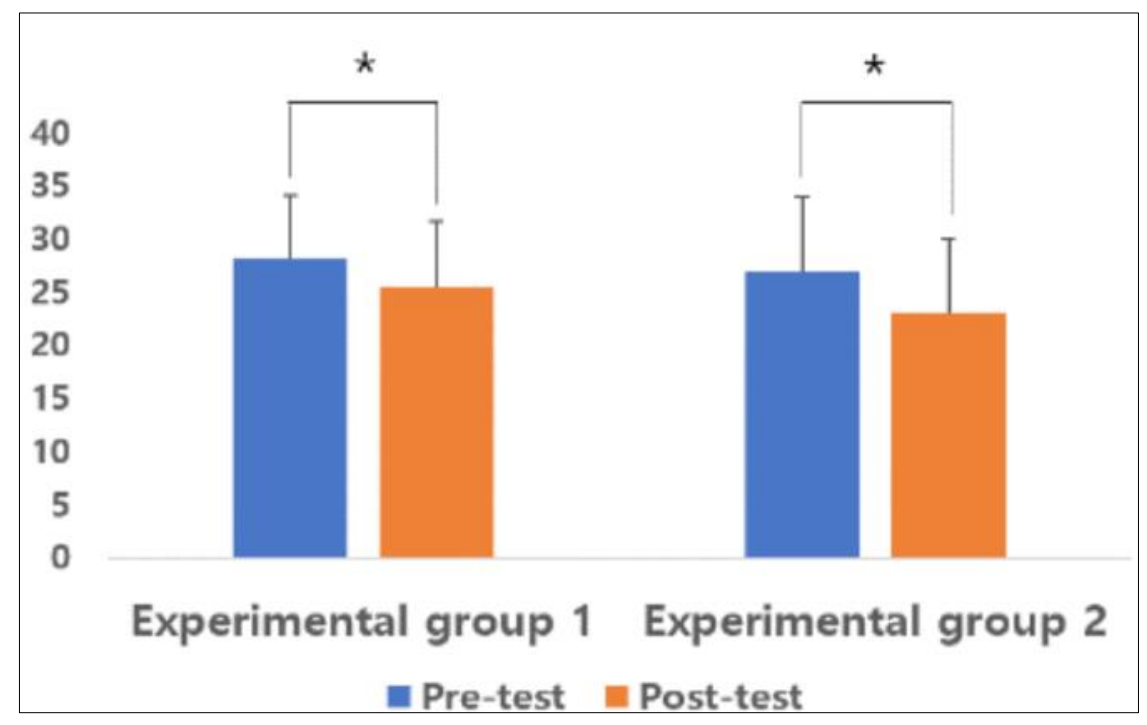

Figure 4 Changes in TUG seconds according to treatment methods

In the BBS test performed to examine the patient's ability to maintain balance, there was a statistically significant difference in both experimental group 1 and experimental group 2 in the post-evaluation compared to the preevaluation. However, there was no statistically significant difference in the comparison of the treatment effect between the experimental groups on the BBS (figure 5).

\section{Discussion}

Stroke is a disease in which blood flow to the brain is blocked due to cerebrovascular disease, heart disease, diabetes, or the like, or a disorder occurs due to bleeding in brain tissue. 1 in 4 stroke patients die within 1 month of acute onset, $9 \%$ recover completely, and even if $73 \%$ recover, problems such as brain lesions, motor impairments, sensory impairment, speech impairment, and cognitive impairment occur [7].

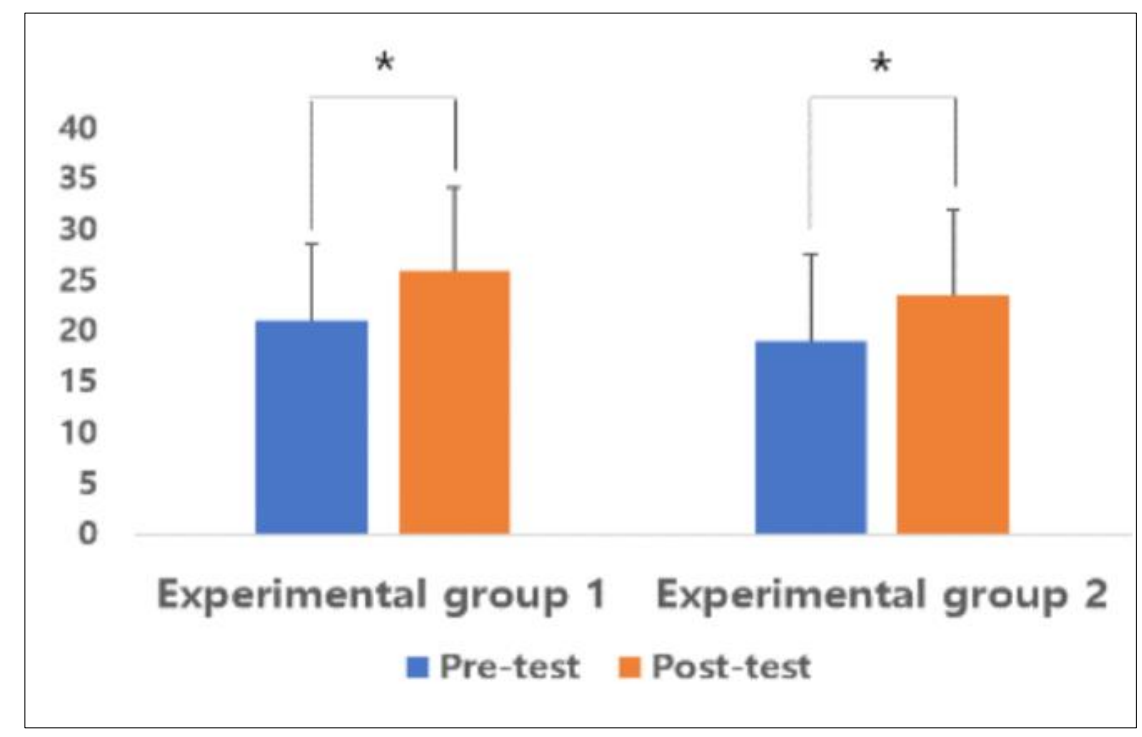

Figure 5 Changes in BBS scores according to treatment methods

In patients with hemiplegia due to stroke, the control ability of the central nervous system on the affected side is lost, the agonist and antagonist muscles are dissonant, excessive muscle tone such as stiffness appears, and proprioception 
and balance are lost. In addition, asymmetric posture, abnormal body balance, reduced ability to carry weight, and loss of certain motor elements that perform delicate functions can cause difficulties in standing or walking [8].

Taping therapy is a treatment method that helps the muscles and joints move while smoothing the extension and contraction of the muscles by wrapping or attaching tape to the joints and muscles of the body.Taping therapy is an intervention method that regulates muscle tension and induces recovery of physical activity for the purpose of inducing efficient physical exercise and restoring function of the body with pain [9]. In addition, a flexible tape is used for the purpose of relieving pain occurring in each part of the human body, preventing various diseases from developing into chronic diseases, and improving obstacles caused by muscle damage [10].

In a study on stroke patients, conservative nervous system physical therapy and kinesio taping were administered 5 times a week for 30 minutes, for a total of 8 weeks. As a result, significant differences were found in ROM and MAS in the nervous system physical therapy group and the taping group. Also, there was a significant difference in the dynamic balance ability in the taping group [11]. In this study, in experimental group 2 to which taping was applied, it was observed that functional activity, gait, and balance ability were statistically improved in the post-evaluation compared to the pre-evaluation.

By applying FES to the peripheral nerve of a patient who is paralyzed due to damage to the upper motor neuron, skeletal muscle contraction can be caused. The reason is that FES is not under the control of the upper nervous system, so it can create functionally useful movements through electrical stimulation of lower motor nerves and muscle fibers that cannot be actively controlled [12].

In the previous study on FES, investigated the possibility of an experiment to prevent foot drop during walking through treadmill training with functional electrical stimulation in 7 stroke patients. The training was conducted 3 times a week for a total of 2 weeks, and the evaluation was evaluated whether functional electrical stimulation was helpful to the person using a video gait analysis, manual strength evaluation, stiffness evaluation, and a questionnaire after the experiment was completed. 3 out of 7 people improved their walking ability, and 6 out of 7 people reported that functional electrical stimulation was helpful for gait training [13]. In this study, the effect of functional electrical stimulation therapy on functional activity, gait, and balance ability of stroke patients were investigated. As a result of the study, statistically significant improvements in functional activity, gait, and balance ability were observed in experimental group 1 to which functional electrical stimulation was applied.

\section{Conclusion}

This study was conducted to investigate the effects of functional electrical stimulation therapy and taping therapy, which can increase proprioception, on functional activity, gait, and balance in stroke patients. As a result of this study, it was confirmed that the functional activity, gait, and balance of stroke patients were statistically increased by functional electrical stimulation treatment and taping treatment.

In other words, it was found that by applying functional electrical stimulation therapy and taping therapy that can increase proprioception to stroke patients, it can have a positive effect on daily activities by increasing functional activity, gait, and balance ability.

\section{Compliance with ethical standards}

\section{Acknowledgments}

This work was supported by the National Foundation of Korea (NRF) grant funded by the Korea government (MSIT) (No-2019R1F1A1057731).

\section{Disclosure of conflict of interest}

No conflict of interest.

\section{Statement of informed consent}

Informed written consent were obtained from all individual participants included in the study. 


\section{References}

[1] Caprio FZ, Sorond FA. Cerebrovascular Disease: Primary and Secondary Stroke Prevention. Med Clin North Am. 2019; 103(2): 295-308.

[2] Barthels D, Das H. Current advances in ischemic stroke research and therapies. Biochim Biophys Acta Mol Basis Dis. 2020; 1866(4): 165260.

[3] Hornby TG, Reisman DS, Ward IG, Scheets PL, Miller A, Haddad D, et al. Clinical Practice Guideline to Improve Locomotor Function Following Chronic Stroke, Incomplete Spinal Cord Injury, and Brain Injury. J Neurol Phys Ther JNPT. 2020; 44(1): 49-100.

[4] Jan S, Arsh A, Darain H, Gul S. A randomized control trial comparing the effects of motor relearning programme and mirror therapy for improving upper limb motor functions in stroke patients. JPMA J Pak Med Assoc. 2019; 69(9): 1242-1245.

[5] Dyer S, Mordaunt DA, Adey-Wakeling Z. Interventions for Post-Stroke Shoulder Pain: An Overview of Systematic Reviews. Int J Gen Med. 2020; 13: 1411-1426.

[6] Huang YC, Chen PC, Tso HH, Yang YC, Ho TL, Leong CP. Effects of kinesio taping on hemiplegic hand in patients with upper limb post-stroke spasticity: a randomized controlled pilot study. Eur J Phys Rehabil Med. 2019; 55(5): 551-557.

[7] Smith EE, Saposnik G, Biessels GJ, Doubal FN, Fornage M, Gorelick PB, et al. Prevention of Stroke in Patients With Silent Cerebrovascular Disease: A Scientific Statement for Healthcare Professionals From the American Heart Association/American Stroke Association. Stroke. 2017; 48(2): e44-71.

[8] Park HK, Lee HJ, Lee SJ, Lee WH. Land-based and aquatic trunk exercise program improve trunk control, balance and activities of daily living ability in stroke: a randomized clinical trial. Eur J Phys Rehabil Med. 2019; 55(6): 687-694.

[9] Ortiz Ramírez J, Pérez de la Cruz S. Therapeutic effects of kinesio taping in children with cerebral palsy: a systematic review. Arch Argent Pediatr. 2017; 115(6): e356-361.

[10] Kim BR, Kang TW. The effects of proprioceptive neuromuscular facilitation lower-leg taping and treadmill training on mobility in patients with stroke. Int J Rehabil Res Int Z Rehabil Rev Int Rech Readaptation. 2018; 41(4): 343-348.

[11] Liao LY, He XH, Li XZ, Ge YL, Gao Q. Effects of kinesiology taping on trunk function, balance, and mobility in stroke patients: a pilot feasibility study. J Phys Ther Sci. 2020; 32(6): 359-364.

[12] Sharif F, Ghulam S, Malik AN, Saeed Q. Effectiveness of Functional Electrical Stimulation (FES) versus Conventional Electrical Stimulation in Gait Rehabilitation of Patients with Stroke. J Coll Physicians Surg-Pak JCPSP. 2017; 27(11): 703-706.

[13] Johnston TE, Keller S, Denzer-Weiler C, Brown L. A Clinical Practice Guideline for the Use of Ankle-Foot Orthoses and Functional Electrical Stimulation Post-Stroke. J Neurol Phys Ther JNPT. 2021; 45(2): 112-196. 